\title{
Melanoma oral: Importancia de un diagnóstico temprano. Un caso clínico.
}

\author{
Oral melanoma: \\ Importance of an early diagnosis. A case report.
}

\author{
Sebastián Zapata ${ }^{1 *}$, Lorena Núñez ${ }^{1}$, Gabriel Zamorano1, Julio Villanueva ${ }^{1,2}$
}

\begin{abstract}
1. Departamento de Cirugía Bucomaxilofacial, Universidad de Chile, Santiago, Chile.

2. Hospital Clínico San Borja Arriarán, Santiago, Chile.
\end{abstract}

Trabajo recibido el 31/12/2015. Aprobado para su publicación el 20/10/2016

* Correspondencia autor: Correo Electrónico: szapatabaeza@gmail.com | Dirección postal: Volcán Llaima 210, Quilicura, Santiago de Chile | Código postal: 8730259 | Teléfono: +56989037382

\section{RESUMEN}

Las lesiones pigmentadas malignas en la mucosa oral son de escasa frecuencia, pero de altas tasas de morbilidad y mortalidad, siendo de mal pronóstico a pesar de agresivos tratamientos que pueden combinar grandes resecciones quirúrgicas con quimioterapia y radioterapia. Dado este escenario, la conducta clínica asociada a la pesquisa de lesiones pigmentadas es la biopsia inmediata para la confirmación o descarte de patología maligna y con ello un inicio temprano de tratamiento. Este artículo trata de una paciente recibida en un hospital público aproximadamente seis meses posteriores a las primeras manifestaciones de una lesión pigmentada en mucosa maxilar, que luego de confirmado el diagnóstico de una lesión maligna fue derivada y manejada por un equipo oncológico del mismo centro.

PALABRAS CLAVE

Melanoma oral; lesiones pigmentada; mucosa oral; patología oral.

Rev. Clin. Periodoncia Implantol. Rehabil. Oral Vol. 10(2); 90-92, 2017.

\section{ABSTRACT}

Pigmented malignant oral mucosa lesions are infrequent, but with high rates of morbidity and mortality, and have a poor prognosis despite aggressive treatments that can combine large surgical resection with chemotherapy and radiotherapy. Given this scenario, the clinical conduct associated with pigmented lesions is the immediate biopsy to confirm or rule out the disease and thus the early initiation of treatment. This article is about a patient attended in a public hospital about six months after the first signs of an injury to maxillary mucosa, which then confirmed the diagnosis of a malignant lesion, who was referred and treated by a cancer team of the same center.

KEY WORDS

Oral melanoma; pigmented lesions; oral mucosa; oral pathology.

Rev. Clin. Periodoncia Implantol. Rehabil. Oral Vol. 10(2); 90-92, 2017.

\section{INTRODUCCIÓN}

Las lesiones pigmentadas en la mucosa oral pueden ser clasificadas en melanóticas y no melanóticas, siendo las primeras más frecuentes. Dentro de las pigmentaciones melanóticas podemos encontrar difusas y focales, las cuales pueden ser fisiológicas o malignas, siendo estas últimas menos frecuentes, pero de gran morbilidad y mortalidad(1), implicando malos pronósticos a pesar de la agresividad de los tratamientos resectivos en conjunto con quimio y radioterapia utilizados para su manejo(2).

Las lesiones pigmentadas pueden corresponder a aumentos de la producción de melanina, como en las pigmentaciones fisiológicas, pigmentaciones post-inflamatorias y máculas melanóticas, más frecuentes en personas de piel oscura; como también al aumento en la multiplicación celular de los melanocitos, como en el caso de los nevos melanocíticos y nevos azules. Estas últimas lesiones deben ser biopsiadas para realizar el diagnóstico diferencial con el melanoma, a diferencia de las primeras, las cuales pueden ser diagnosticadas a partir de la clínica de cada paciente en particular. Existen también otras lesiones pigmentadas entre las que se cuentan: Melanosis del fumador, relacionada al hábito tabáquico, tanto en su génesis como en su probable regresión al suspender el hábito; melanoacantoma oral, aparentemente de origen reaccional, requiriendo biopsia para su diagnóstico; tatuaje de amalgama, principalmente debido al contacto de las mucosas con obturaciones realizadas con este material, lo cual hace necesaria una acuciosa anamnesis y examen físico para su diagnóstico; pigmentaciones por drogas, como arsénico, clotrimazol, tetraciclinas, etc.; relacionadas al Síndrome de Peutz-Jeghers, en conjunto con poliposis intestinal y aumento de probabilidad de carcinomas del tracto digestivo, mama y tiroides. $^{(3)}$

Los melanomas constituyen la proliferación descontrolada de melanocitos a nivel de la unión entre el epitelio y el tejido conjuntivo, invadiendo este último a medida que avanza en su crecimiento(3). Corresponden aproximadamente al $1-3 \%$ de los tumores malignos ${ }^{(4)}$, constituyendo el melanoma oral el $1 \%$ de ellos, el cual a su vez significa el $0,5 \%$ de todas las lesiones malignas de la cavidad oral ${ }^{(2)}$. Lo anterior da cuenta de la baja frecuencia con que este tipo de lesiones malignas afectan la cavidad oral, sin embargo debe estar presente entre los diagnósticos diferenciales de los clínicos, dadas las implicancias terapéuticas y pronosticas que posee. El lugar más común de aparición del melanoma oral es el paladar, con un $40 \%$ de los casos, presentándose como una mancha café o negra de crecimiento lento, o bien, un aumento de volumen pigmentado, de rápida progresión, ulcerada, sangrante con dolor y destrucción ósea asociada ${ }^{(3)}$. 
Existe controversia sobre el método de biopsia a utilizar cuando se sospecha de este tipo de lesiones, dada la probabilidad de producir diseminación de células neoplásicas al practicar una biopsia incisional. Sin embargo, existen estudios en los cuales se ha analizado este dilema y que no han vislumbrado diferencias en cuanto a dispersión del proceso neoplásico con uno u otro procedimiento ${ }^{(2,5)}$. En lo que existe consenso es que ante la sospecha, debe realizarse, de una u otra forma, un diagnóstico en el menor tiempo posible.

El pronóstico de los pacientes con este tipo de lesiones malignas se relaciona directamente con el grado de invasión y diseminación de las células que la componen. La estadificación del melanoma está descrita principalmente para lesiones cutáneas, sin embargo, se puede realizar un paralelo aproximado en lesiones de la mucosa oral, siendo hoy en día la clasificación del American Joint Comitee on Cancer (AJCC) la más utilizada, la cual considera el espesor del tumor según los criterios clásicos de Breslow descritos en los años '70, en conjunto con el grado de ulceración de la lesión y su tasa de mitosis celular para definir la variable T de la clasificación TNM, siendo una de las variables más importantes para establecer los procedimientos terapéuticos necesarios y el pronóstico de sobrevida de estos pacientes. ${ }^{(6)}$

El tratamiento definitivo del melanoma mucoso es combinación de la resección completa de la lesión con márgenes de seguridad, que en el caso de la mucosa oral se ha consensuado en alrededor de $1,5 \mathrm{~cm}^{(2)}$, y también puede ser complementado con quimio, radio e inmunoterapia, aunque ninguna de ellas ha demostrado aumentar de manera significativa el período de vida libre de cáncer post terapéutico, pero se continúan utilizando con el fin de poder brindar al paciente un eventual mejor pronóstico, ya que pueden haber recurrencias incluso frente a un tumor resecado con grandes márgenes de seguridad ${ }^{(1,2,7)}$.

En cuanto al pronóstico, la sobrevivencia de estos pacientes a 3 y 5 años no supera el $43 \%$, siendo este último el valor correspondiente a pacientes diagnosticados a temprana edad. La cirugía resectiva mejora el pronóstico y aún más si se acompaña por disección de ganglios cervicales y alguna terapia complementaria, ya sea quimio, radio o inmunoterapia(8)(9). Por otro lado, es importante mencionar que el diagnóstico acucioso también influye bastante en el pronóstico que puede entregarse al paciente, ya que pacientes en estadio I, es decir, sin invasión de tejidos subyacentes o circundantes, tienen mejor pronóstico que aquellos en estados II y III, que respectivamente incluyen la invasión de la lámina propia y la de tejidos profundos ${ }^{(10)}$.

El objetivo del presente artículo es mostrar un caso clínico de una lesión pigmentada, enfatizado principalmente en su proceso de diagnóstico.

\section{CASO CLÍNICO}

Se presenta en el Servicio de Cirugía Maxilofacial del Hospital San Borja-Arriarán de Santiago de Chile una paciente femenina de 47 años de edad, hipertensa y depresiva controlada con fármacos, consultando derivada desde consultorio por lesión pigmentada en maxilar superior derecho.

En cuanto a la anamnesis, la paciente relata haber sido atendida en una clínica universitaria para fines protésicos, en donde hace seis meses aproximadamente comienza a manifestarse la lesión, sin haberse realizado un diagnóstico diferencial de la misma, experimentando en el último tiempo crecimiento y sangramiento, especialmente al cepillado de los dientes con los que guarda relación.

Al examen clínico se observa aumento de volumen pigmentado en maxilar superior derecho, $2 \mathrm{~cm}$ de diámetro mayor aproximadamente, en la zona molar, asintomática, de color morado-negruzco, de consistencia firme y con manifestación tanto en zona vestibular como palatina de los dientes comprometidos, con adenopatía submandibular y cervical bilateral asociada. Al examen radiográfico no se observan manifestaciones de la lesión en tejidos duros.

Se realiza biopsia incisional de la porción vestibular de la lesión, la cual arroja el diagnóstico definitivo de melanoma en submucosa oral (S100+/HMB45+).

Finalmente la paciente es derivada a Oncología para manejo definitivo de la lesión donde se decide la realización de una maxilectomía parcial, con vaciamiento de la cadena ganglionar cervical y radioterapia adjunta, para lo cual es enviada nuevamente al servicio de cirugía maxilofacial para la eliminación de focos orales previo a dicho tratamiento. Paralelamente, la paciente se encuentra bajo manejo kinesiológico de sus cicatrices y dinámica facial asociada. Posterior a su radioterapia la paciente será derivada a rehabilitación protésica para la recuperación funcional y estética.

\section{DISCUSIÓN}

A la luz de los antecedentes expuestos y teniendo en cuenta este
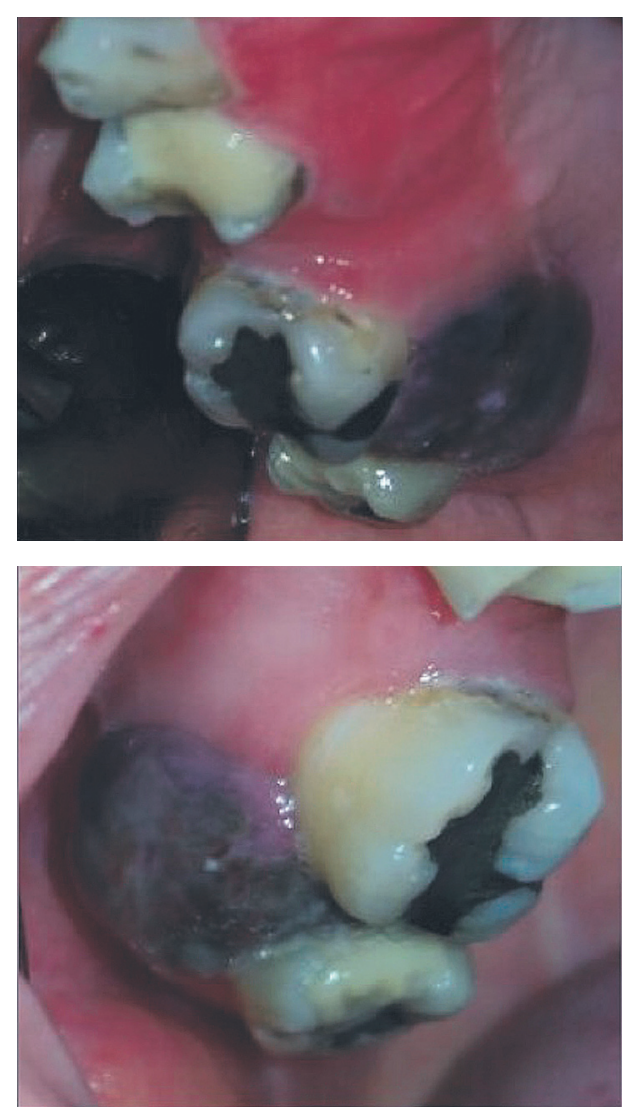

$\overline{\text { Figura 1. Visiones vestibular y palatina de lesión pigmentada en maxilar }}$ superior derecho.

caso, resalta la importancia del examen clínico y la sospecha inicial sobre lesiones o condiciones que se escapen a la normalidad de los tejidos orales. En este caso, la paciente recibió atención odontológica por al menos seis meses, en los cuales la lesión experimentó crecimiento y aparición de signos de alerta, como el sangrado al cepillado, sobre los que no se puso la suficiente atención ${ }^{(6)}$. Esto ultimo nos alerta sobre la precaución que como profesionales debemos tener al enfrentarnos a la clínica, donde el simple acto de observar detenidamente las estructuras orales puede hacer la diferencia al diagnosticar una lesión como esta tempranamente.

Sin duda el pronóstico de los pacientes con melanoma mejora en cuanto la pesquisa de las lesiones es temprana y las lesiones aún no han invadido la lámina basal y los tejidos subyacentes, pudiéndose practicar biopsias excisionales sin el riesgo latente de provocar la dispersión de las células malignas a otros territorios ${ }^{(2,5)}$ y sin duda, sin tener que realizar grandes resecciones de tejidos que implican altos costos funcionales y emocionales para los pacientes.

La prevención primaria de las lesiones melanóticas tiene que ver con la educación a la población para reducir el riesgo de desarrollarlas, sin embargo esto es mucho más simple de aplicar en el caso de los melanomas cutáneos, dados los factores de riesgo manejables, como por ejemplo la exposición a los rayos UV y el uso de bloqueadores solares $^{(11)}$. Lo anterior, en el caso del melanoma oral no está bien identificado, por lo que es difícil apuntar a blancos específicos en la prevención primaria. Por otro lado, la detección precoz de lesiones sí es un punto importante en lesiones orales, lo cual debe ser practicado por los profesionales odontólogos y al mismo tiempo debemos educar a nuestra población para realizarse autoxamen de manera regular, lo cual en el caso del melanoma cutáneo ha mostrado mejorar en gran medida el pronóstico y las morbilidades asociadas a esta enfermedad ${ }^{(12)}$. Lo anterior está relacionado directamente con el establecimiento de políticas públicas de salud que apunten en este mismo sentido y no principalmente a subsanar los déficit en términos terapéuticos y rehabilitadores de los pacientes que sufren esta patología.

Finalmente, la principal conclusión de este caso es que está en nuestras manos como clínicos hacer frente a patologías poco frecuentes, pero tan costosas en todo sentido para los pacientes y para el sistema de salud como la presentada en este artículo. Lo anterior es posible mediante conductas que debiesen formar parte del examen clínico 

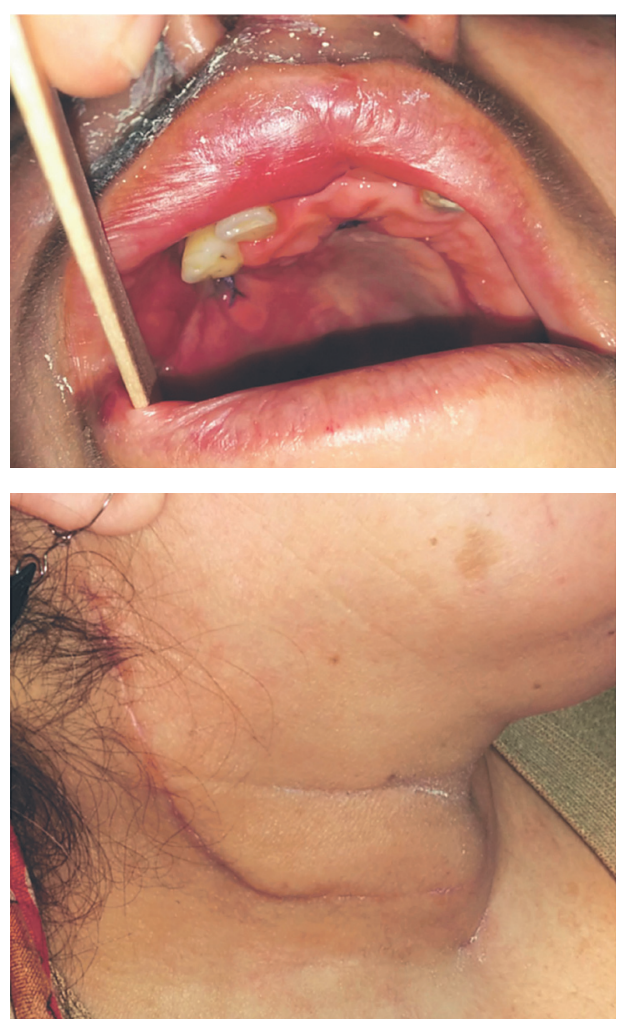

Figura 2. Imágenes posteriores a maxilectomía parcial y vaciamiento cervical. habitual, tales como un examen visual acucioso de todas las áreas de la mucosa oral, con el fin de propender a un diagnóstico precoz de lesiones potencialmente malignas y su tratamiento oportuno, con el fin de brindar un mejor pronóstico y calidad de vida para los pacientes afectados.

\section{RESPONSABILIDADES ÉTICAS}

Protección de personas y animales. Los autores declaran que para esta investigación no se han realizado experimentos en seres humanos ni en animales.

Confidencialidad de los datos. Los autores declaran que han seguido los protocolos de su centro de trabajo sobre la publicación de datos de pacientes.

Derecho a la privacidad y consentimiento informado. Los autores han obtenido el consentimiento informado de los pacientes y/o sujetos referidos en el artículo. Este documento obra en poder del autor de correspondencia.

\section{Bibliografía}

1. Mohan M, Sukhadia VY, Pai D, Bhat S. Oral malignant melanoma: systematic review of literature and report of two cases. Oral Surg Oral Med Oral Pathol Oral Radiol. 2013 Oct;116(4):e247-54

2. Meleti M, Leemans CR, Mooi WJ, Vescovi P, van der Waal I. Oral malignant melanoma: a review of the literature. Oral Oncol. 2007 Feb;43(2):116-21.

3. Gondak R-O, da Silva-Jorge R, Jorge J, Lopes M-A, Vargas P-A. Oral pigmented lesions: Clinicopathologic features and review of the literature. Med Oral Patol Oral Cir Bucal. 2012 Nov; 17(6):e919-24.

4. Parkin DM, Bray F, Ferlay J, Pisani P. Global cancer statistics, 2002. CA Cancer J Clin. 2005 Apr:55(2):74-108.

5. Kruse ALD, Riener MO, Graetz KW, Luebbers H-T. Mucosal malignant melanomas in head and neck surgery: a retrospective study of six patients and review of the literature. Oral Maxillofac Surg. 2010 Sep;14(3):143-7.

6. Boland GM, Gershenwald JE. Principles of Melanoma Staging. Cancer Treat Res. 2016;167:131-48.
7. Seigler HF. Mucosal melanoma. J Surg Oncol. 2004 Jul 1;86(4):187-8.

8. Sun C-Z, Chen Y-F, Jiang Y-E, Hu Z-D, Yang A-K, Song M. Treatment and prognosis of oral mucosal melanoma. Oral Oncol. 2012 Jul;48(7):647-52.

9. Zhu H, Dong D, Li F, Liu D, Wang L, Fu J, et al. Clinicopathologic features and prognostic factors in patients with non-cutaneous malignant melanoma: a singlecenter retrospective study of 71 cases. Int J Dermatol. 2015 Dec;54(12):1390-5.

10. Mücke T, Hölzle F, Kesting MR, Loeffelbein DJ, Robitzky LK, Hohlweg-Majert $B$, et al. Tumor size and depth in primary malignant melanoma in the oral cavity influences survival. J Oral Maxillofac Surg 2009 Jul;67(7):1409-15.

11. Tripp MK, Watson M, Balk SJ, Swetter SM, Gershenwald JE. State of the science on prevention and screening to reduce melanoma incidence and mortality: The time is now. CA Cancer J Clin. 2016 May 27; doi: 10.3322/caac.21352. (Epub ahead of print)

12. De Braud F, Khayat D, Kroon BBR, Valdagni R, Bruzzi P, Cascinelli N. Malignant melanoma. Crit Rev Oncol Hematol. 2003 Jul;47(1):35-63. 